\title{
Multi-organ spreading of Actinobacillus pleuropneumoniae serovar 7 in weaned pigs during the first week after experimental infection
}

\author{
Doris Hoeltig ${ }^{1^{*}} \mathbb{D}$, Judith Rohde ${ }^{2}$, Renate Frase ${ }^{3}$, Florian Nietfeld ${ }^{4}$, Karl-Heinz Waldmann ${ }^{1}$, \\ Peter Valentin-Weigand ${ }^{2}$ and Jochen Meens ${ }^{2}$
}

\begin{abstract}
Actinobacillus (A.) pleuropneumoniae is normally considered strictly adapted to the respiratory tract of swine. Despite this, scattered case reports of arthritis, osteomyelitis, hepatitis, meningitis or nephritis exist, in which A. pleuropneumoniae remained the only detectable pathogen. Therefore, the aim of this study was to investigate whether spreading to other organs than the lungs is incidental or may occur more frequently. For this, organ samples (blood, liver, spleen, kidney, tarsal and carpal joints, meninges, pleural and pericardial fluids) from weaners $(n=47)$ infected experimentally with A. pleuropneumoniae serovar 7 by aerosol infection (infection dose: $10.9 \times 10^{3} \mathrm{cfu} / \mathrm{animal}$ ) were examined by culture during the first week after infection. In addition, tissue samples of eight weaners were examined by histology and immunohistochemistry (IHC). A. pleuropneumoniae was isolated in all examined sample sites (86.7\% pleural fluids, $73.3 \%$ pericardial fluids, $50.0 \%$ blood, $61.7 \%$ liver, $51.1 \%$ spleen, $55.3 \%$ kidney, $14.9 \%$ tarsal joints, $12.8 \%$ carpal joints, $27.7 \%$ meninges). These results were also obtained from animals with only mild clinical symptoms. IHC detection confirmed these findings in all locations except carpal joints. Histological examination revealed purulent hepatitis $(n=2)$, nephritis $(n=1)$ and beginning meningitis $(n=2)$. Isolation results were significantly correlated $(p<0.001)$ with the degree of lung colonization and, to a lower extent, with the severity of disease. Detection of A. pleuropneumoniae in peripheral tissues was significantly correlated to spleen colonization. In conclusion, multi-organ spreading of $A$. pleuropneumoniae serovar 7 strain AP 76 seems to occur more frequently during acute infection following effective lung colonization than previously thought.
\end{abstract}

\section{Introduction}

Actinobacillus (A.) pleuropneumoniae, a Gram-negative bacterium belonging to the family Pasteurellaceae, is one of the most important respiratory tract pathogens in the pig industry. A. pleuropneumoniae is distributed worldwide and considered a primary pathogen that can cause severe respiratory disease. It affects the animal's welfare due to peracute to chronic diseases, including severe

\footnotetext{
*Correspondence: doris.hoeltig@tiho-hannover.de

${ }^{1}$ Clinic for Swine and Small Ruminants and forensic Medicine and Ambulatory Service, University of Veterinary Medicine, Foundation, Bischofsholer Damm 15, 30173 Hannover, Germany

Full list of author information is available at the end of the article
}

fibrino-haemorrhagic and necrotizing pleuropneumonia with an increase in mortality [1,2]. Therefore, it leads to severe economic losses in the pig industry which are due to the loss of animals, and costs of disease treatment as well as reduced performance during the chronic course of disease resulting in a prolonged fattening period of the animals $[3,4]$. The severity of disease depends on several factors, including the infecting serovar, infection dose, co-infections, immune status and genetic background of the animal, as well as environmental factors [5-7]. In addition to its primary role in porcine pleuropneumonia $A$. pleuropneumoniae is also often involved in the development of the porcine respiratory disease complex, a co-infection of the respiratory tract of pigs [8-10]. In 
contrast to certain other porcine respiratory pathogens, e.g., Haemophilus (H.) parasuis [10, 11], A. pleuropneumoniae is considered a non-invasive lung pathogen [12] which solely affects lungs and pleura by spreading from the infected lung to the pleura via lymph vessels or oedematous fluid [13]. A bacteraemia is very rare [12, 13].

Despite its non-invasive character there are several case reports about non-respiratory clinical diseases in which A. pleuropneumoniae was the only detectable pathogen. These reports include cases of fibrino-purulent arthritis and necrotizing osteomyelitis [14], granulomatous hepatitis [15], meningitis and, nephritis [16] as well as endocarditis and fibrinous peritonitis [17]. These recurring case reports prompted us to find out if A. pleuropneumoniae can be detected more frequently within different body tissues during the acute phase of disease. For this, we investigated possible spreading of A. pleuropneumoniae to body tissues other than the lungs in experimentally infected weaners.

\section{Materials and methods Animals and housing conditions}

To investigate the possible spreading of A. pleuropneumoniae to other organs than lungs forty-seven weaners experimentally infected with A. pleuropneumoniae serovar 7 from another infection trial were chosen by simple randomization. This infection trial was originally conducted in the context of evaluation of genetically determined susceptibility of pigs towards the pathogen. The pigs were kept in accordance with the Guidelines for Protection of Vertebrate Animals used for experimental and other Scientific Purposes, European Treaty Series, nos. $123 / 170$. Study setup and housing of the animals were approved by an independent local committee on ethics. The pigs were fed a commercial standardized diet and were provided $2.5 \mathrm{~kg}$ of hay flakes per group $\left({ }^{\circledR} \mathrm{AGROBS}\right.$ Pre Alpin Wiesenflakes, Co. AGROBS, Degerndorf, Germany) per day as material for rooting and manipulation. The group size was 8 to 10 pigs on $8 \mathrm{~m}^{2}$. Ambient temperature was $27.5^{\circ} \mathrm{C} \pm 1.9^{\circ} \mathrm{C}(\mathrm{MEAN} \pm \mathrm{SD})$ and humidity was $33.2 \% \pm 12.5 \%(\mathrm{MEAN} \pm \mathrm{SD})$. Within each pen $2.8 \mathrm{~m}^{2}$ were covered with a rubber mat, heated by two infrared lamps, for bedding area. Allocation to the housing groups was also performed by simple randomization. Drinking quality water was constantly available. The pigs arrived 3 weeks prior to infection for an adequate adaptation to diet and new environment.

\section{Experimental infection}

Prior to experimental infection all pigs underwent a general clinical examination and bronchoalveolar lavage fluids (100 mL 0.9\% NaCl-solution ad us. vet., Co. WDT,
Garbsen, Germany) as well as serum samples were taken. The bronchoalveolar lavage fluid was examined by bacteriological culture and PCR [18] for the absence of A. pleuropneumoniae. Bronchoalveolar lavage was performed under general anesthesia with $20 \mathrm{mg} / \mathrm{kg}$ Ketamine i.m. (Ketamin $100 \mathrm{mg} / \mathrm{mL}^{\circledR}$, Co. CP-Pharma, Burgdorf, Germany) and $2 \mathrm{mg} / \mathrm{kg}$ Azaperon i.m. (Stresnil ${ }^{\mathbb{B}}$, Co. Janssen-Cilag $\mathrm{GmbH}$, Baar, Switzerland). Serum samples were analyzed by ApxIV-ELISA (IDEXX APP-ApxIV Ab Test $^{\circledR}$, Co. IDEXX Laboratories, Maine, USA). Only pigs confirmed as clinically healthy and negative for A. pleuropneumoniae by direct and indirect screening procedures were included for infection. At the time of infection the pigs were 7 weeks of age and had a mean bodyweight of $12.6 \mathrm{~kg} \pm 2.1 \mathrm{~kg}$.

The experimental infection was done by aerosol infection with a total exposure time of $30 \mathrm{~min}$ [6]. For this, approximately $1 \times 10^{5}$ bacteria of $A$. pleuropneumoniae AP76 serovar 7 were nebulized resulting in an aerosol concentration of $1 \times 10^{2}$ colony forming units (cfu) per liter aerosol. Based upon a mean tidal volume of pigs of $9 \mathrm{~mL} / \mathrm{kg} \mathrm{BW}[19]$ the mean infection dose inhaled per pig was $10.9 \times 10^{3} \mathrm{cfu}$. The group size for infection was five to six pigs. After infection the pigs were clinically monitored every $2 \mathrm{~h}$ within the first $48 \mathrm{~h}$ after infection. Subsequently, the clinical examination was done twice a day. Exit criteria for euthanasia of the pigs were defined to minimize the suffering of the infected animals. Exit criteria were as follows: a respiratory score (Table 1) of 3; a depression score (Table 1) of 3; a rectal body temperature $>42.0^{\circ} \mathrm{C}$; a respiratory or depression score greater than 1 and a body temperature $<37.5^{\circ} \mathrm{C}$; a body temperature $>40.3^{\circ} \mathrm{C}$ and/or a depression score and respiratory score $>1$ for more than two consecutive days; a body temperature $>40.3{ }^{\circ} \mathrm{C}$ and/or depression score and respiratory score $>0$ for more than two consecutive days from day three post infection onwards; any unpredictable event, reaction to treatment or disease leading to a moderate reduction of general condition or inducing pain for more than $48 \mathrm{~h}$; any unpredictable event, reaction to treatment or disease leading to severe reduction of general condition or inducing pain.

\section{Clinical investigation}

The monitoring of the clinical signs consisted of an assessment of posture, behavior, feed intake, rectal body temperature, vomiting, breathing noise, dyspnea, respiratory frequency, coughing and skin color. The results of the clinical examinations were transferred to an objective clinical scoring system [20]. According to this scoring system the pigs were assessed as non-diseased when showing scoring points $\leq 1.3$; as mildly diseased when showing scoring points $>1.3 \leq 12.5$; as moderately 
Table 1 Scoring systems used for the application of exit criteria

\begin{tabular}{|c|c|c|c|c|}
\hline \multirow[t]{2}{*}{ Scoring systems } & \multicolumn{4}{|c|}{ Scoring scale based upon clinical symptoms } \\
\hline & 0 & 1 & 2 & 3 \\
\hline Respiratory score & $\begin{array}{l}\text { No clinical } \\
\text { signs of } \\
\text { respiratory } \\
\text { disease }\end{array}$ & $\begin{array}{l}\text { Breathing frequency of } 35-55 / \\
\text { min and/or occasional cough- } \\
\text { ing }\end{array}$ & $\begin{array}{l}\text { Breathing frequency of } 56-90 / \mathrm{min} \text { and/ } \\
\text { or multiple coughing periods within } \\
10 \text { min and dyspnea }\end{array}$ & $\begin{array}{l}\text { Breathing frequency }>90 / \text { min and } \\
\text { cyanosis or gasping or open- } \\
\text { mouth breathing or breathing } \\
\text { frequency }>90 / \text { min and cyanosis and } \\
\text { gasping or open-mouth breathing }\end{array}$ \\
\hline Depression score & $\begin{array}{l}\text { Active, alert, } \\
\text { normal } \\
\text { ingestion }\end{array}$ & Calm, alert, decreased ingestion & $\begin{array}{l}\text { Dull, increased recumbence, increased } \\
\text { reaction time, still moving to the feed- } \\
\text { ing trough but without or only small } \\
\text { feed intake or dull, sitting like a dog, } \\
\text { increased reaction time, still moving } \\
\text { to the feeding trough but without or } \\
\text { only small feed intake }\end{array}$ & $\begin{array}{l}\text { Apathetic, no reaction to stimulation } \\
\text { and/or shaky movements without } \\
\text { lying down and/or standing with } \\
\text { head down without lying down and/ } \\
\text { or vomiting and/or foam around } \\
\text { nostrils and mouth }\end{array}$ \\
\hline
\end{tabular}

diseased with scoring points $>12.5 \leq 23.7$ and as severely diseased when reaching scoring points $>23.7$ on day seven post-infection (pi).

\section{Necropsy and bacteriological examination}

Seven days post infection (or earlier in case of withdrawal due to exit criteria) the pigs were euthanized by intravenous application of $80 \mathrm{mg} / \mathrm{kg}$ pentobarbital (Euthadorm $^{\circledR}$, Co. CP Pharma GmbH, Burgdorf, Germany). Necropsy was performed and the degree of lung lesions was assessed using a lung lesion score [21]. For this score a schematic map of the lung is divided into triangles. According to the assessed lung lesions triangles are marked. Each lung lobe can reach a maximum score of 5 resulting in an overall maximum score of 35 . The degree of lung lesions was classified as mild with scoring points $>0 \leq 11.6$; as moderate with scoring points $>11.6 \leq 23.2$ and as severe when reaching scoring points $>23.2$

For the isolation of $A$. pleuropneumoniae tissue samples were taken from 13 different locations. In total seven lung tissue samples of defined positions located in the outer third of each of the seven lung lobes, three central organ samples including liver, spleen and left kidney and three peripheral tissue samples including swabs from the meninges as well as the carpal and tarsal joints of the animals were collected. All tissue samples had a size of approximately $1 \mathrm{~cm}^{2}$. In cases where there were no macroscopic lesions in the defined locations of the lung lobes but in other parts of the lung lobes additional samples from the macroscopically altered regions were taken. Tissue samples and swab samples were plated on A. pleuropneumoniae-selective blood agar [22] using the quadrant streaking method. Abundance of growth was assessed semi-quantitatively. Additionally from 15 pigs pleural and pericardial exudates were sampled and evaluated regarding volume and number of viable A. pleuropneumoniae cells. The second was determined by plating tenfold serial dilution on $A$. pleuropneumoniae-selective blood agar. Bacterial isolates were identified as $A$. pleuropneumoniae by PCR amplification of the apxIV gene [23].

The amount of growth for each organ or swab sample was transferred to a scoring system $(0=$ no growth; $1=$ sparse growth; $2=$ moderate growth; $3=$ heavy growth). For the lung tissue samples the level of isolation from the lungs was translated into a combined isolation score [24]. For this purpose the amount of growth from all lung tissue samples was added and divided by the total number of lung tissue samples. Results were classified as low-grade isolation level (score $0-1$ ), moderate ( $>1$ and $\leq 2)$ and high-grade $(>2-3)$.

Additionally blood samples were taken from the Vena jugularis at the time of euthanasia from 26 pigs. These blood samples were inoculated into SIGNAL blood culture systems (SIGNAL blood culture system ${ }^{\circledR}$; Co. Oxoid, Basingstroke, Hampshire, UK) and bottles with medium in the growth indicator device were subcultured for $A$. pleuropneumoniae. From 23 of these pigs $0.5 \mathrm{~mL}$ of the taken blood sample was directly plated on A. pleuropneumoniae-selective blood agar [22] using the quadrant streaking method, too. For these directly plated blood samples the abundance of growth was assessed semiquantitatively like for the other tissue samples.

\section{Histological examination and immunohistochemistry}

A total of eight pigs were chosen randomly for additional histological examination and immunohistochemical (IHC) investigation. Tissues collected at necropsy for these investigations included liver, spleen, pericardium, peritoneum, kidney, cerebrum, and carpal and tarsal synovialis. Tissues were fixed by immersion in $10 \%$ neutral buffered formalin for 1-7 days and routinely processed in an automated tissue processor, embedded in paraffin, sectioned at $3 \mu \mathrm{m}$, and stained with hematoxylin 
and eosin. Additional sections were mounted on polyL-lysine-coated slides (SuperFrost Plus ${ }^{\circledR}$; Thermo Fischer Scientific, Dreieich, Germany) for immunohistochemical evaluation.

A biotin-free horse-radish-peroxidase (HRP)-polymerbased detection system (Ultravison LP HRP, Thermo Fisher Scientific, Dreieich, Germany) was used for immunohistochemical detection of A. pleuropneumoniae antigen. Antigen retrieval was achieved by immersion of the deparaffinized rehydrated slides in preheated EDTA buffer ( $\mathrm{pH}$ 8) for $15 \mathrm{~min}$ at $90{ }^{\circ} \mathrm{C}$ in a steamer. After cooling down to at least $60{ }^{\circ} \mathrm{C}$ the slides were washed 3 times in Tris buffer containing 0.05\% Tween 20 (Carl Roth $\mathrm{GmbH}$, Karlsruhe, Germany) at room temperature. Then they were blocked for $7 \mathrm{~min}$ at room temperature with Ultra V blocking solution (Thermo Fisher Scientific, Dreieich, Germany) and incubated with a mixture of two primary antibodies. These primary antibodies had been produced previously in rabbit using recombinant Outer Membrane Lipoprotein A (OmlA) of A pleuropneumoniae serovar 1 and 5 isolates $[25,26]$. The ability of the anti-OmlA serovar 1 serum to detect $A$. pleuropneumoniae serovars $1-12$ and 14 and the ability of the antiOmlA serovar 5 serum to detect $A$. pleuropneumoniae serovar 5-8 and 10 had been verified previously during western blot validation assays performed at the IVD laboratory using isolates of $A$. pleuropneumoniae serovars $1-15$. No unspecific cross-reaction of the primary antibodies was detected in western blot and dot blot assays with Actinobacillus porcitonsillarum, A. minor, A. porcinus, A. indolicus, A. suis, Haemophilus parasuis or Pasteurella multocida. After primary antibody incubation overnight at $4{ }^{\circ} \mathrm{C}$ the slides were rinsed 3 times in Tris buffer containing $0.05 \%$ Tween 20 (TBS-T) and incubated with the secondary antibody (Primary Antibody Enhancer of the Ultravision Detection LP kit mentioned above) for $15 \mathrm{~min}$ at room temperature. Following 3 washing steps in TBS-T the slides were incubated with the HRP-polymer for $30 \mathrm{~min}$ at room temperature. After 3 washing steps in TBS-T the slides were immersed in 3\% hydrogen peroxide for $10 \mathrm{~min}$ in order to block endogenous peroxidase. Followed by 3 washing steps in TBS-T the slides were treated with 3,3-diaminobenzidine tetrahydrochloride (DAB) chromogen plus substrate solution (Thermo Fisher Scientific, Dreieich, Germany) for $10 \mathrm{~min}$, washed again in TBS-T and counterstained with hematoxylin Gill 2.

\section{Statistical methods}

The collected data were transferred to an Excel ${ }^{\circledR}$ based database (Co. Microsoft Cooperation, Dublin, Ireland). Statistical analyses were carried out using Excel $^{\circledR}$ and IBM SPSS Statistics ${ }^{\circledR}$ (Co. IBM Deutschland GmbH,
Ehningen, Germany). For correlation analysis Spearman Rank Correlations were calculated. Correlations of $<0.05$ were classified as significant and of $<0.01$ as highly significant.

\section{Results}

Based on the clinical scoring, 7 of 47 infected pigs were classified as non-diseased, 21 pigs as mildly diseased, 3 pigs as moderately diseased and 16 pigs as severely diseased. Nineteen pigs had to be euthanized due to acute exit criteria and 1 pig due to chronic exit criteria. Twenty-seven pigs were euthanized on day $7 \mathrm{pi}$. The degree of gross lung lesions was mild in 13 pigs, moderate in 12 pigs and severe in 19 pigs. In 3 pigs there were no macroscopic lung lesions. For the lung tissue, isolation score of A. pleuropneumoniae was low-grade in 9 pigs, moderate in 13 pigs and high-grade in 22 pigs. A. pleuropneumoniae could not be isolated from the lungs of 3 pigs.

An overview of the isolation results from other tissues than the lungs is shown in Table 2. A. pleuropneumoniae could be cultured from $50 \%$ of the blood samples by blood culture, from $43.5 \%$ of the blood samples by direct plating, from $61.7 \%$ of the liver samples, $51.1 \%$ of the spleen samples, $55.3 \%$ of the kidney samples, $14.9 \%$ of the tarsal joints swabs, $12.8 \%$ of the carpal joints swabs and $27.7 \%$ of the meningeal swabs. The pleural fluid was positive for A. pleuropneumoniae in $86.7 \%$ and the pericardial fluid in $73.3 \%$ (Table 3 ). The bacterium could not be reisolated from any of these locations in pigs classified as non-diseased. Within the sampled pleural fluids the volumes ranged from 1 to $230 \mathrm{~mL}$ and the isolation results of these samples ranged from $9.5 \times 10^{3}$ to $1.3 \times 10^{9} \mathrm{cfu}$. The volumes of the pericardial fluids ranged from 1 to $70 \mathrm{~mL}$ and here the isolation results ranged from 2 to $2.0 \times 10^{8} \mathrm{cfu}$. From the tissue samples isolation scores for A. pleuropneumoniae ranged from low-grade to highgrade whereas the isolation score was only low-grade (76.9\%) and moderate (23.1\%) from meningeal swabs and only low-grade (100\%) from tarsal and carpal joint swabs. A. pleuropneumoniae could be cultured from all these locations not only from pigs classified as moderate or severely diseased but also from pigs classified as mildly diseased (blood: 2 pigs; liver: 15 pigs; spleen: 14 pigs; kidney: 13 pigs; tarsal swabs: 7 pigs; carpal swabs: 6 pigs; meningeal swabs: 10 pigs). Isolation was possible from animals euthanized due to exit criteria (blood: 11 pigs; liver: 16 pigs; spleen: 16 pigs; kidney: 14 pigs; tarsal joints: 5 pigs; carpal joints: 5 pigs; meninges: 9 pigs) as well as from animals euthanized 7 days pi (blood: 2 pigs; liver: 13 pigs; spleen: 8 pigs; kidney: 12 pigs; tarsal joints: 2 pigs; carpal joints: 1 pig; meninges: 4 pigs). A. pleuropneumoniae was not detected in organ samples of pigs 


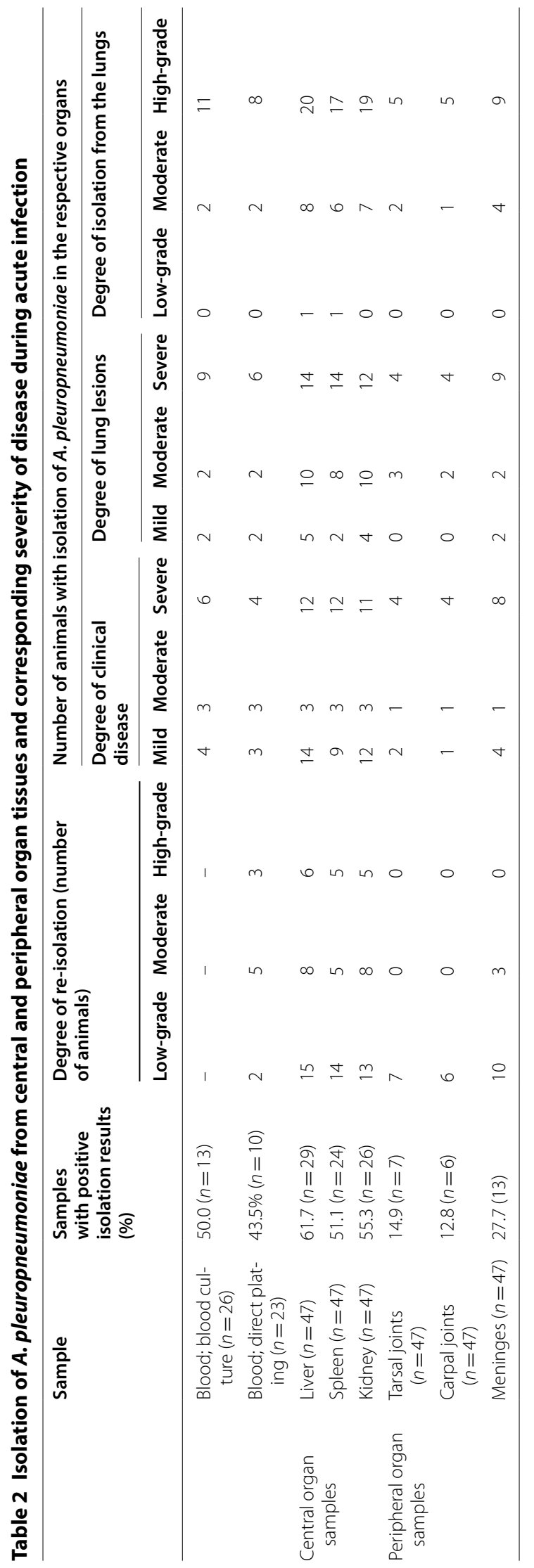


Table 3 Detection of A. pleuropneumoniae in pleural and pericardial fluids and corresponding severity of disease during acute infection

\begin{tabular}{|c|c|c|c|c|c|c|c|c|}
\hline & \multicolumn{4}{|c|}{ Degree of clinical disease } & \multicolumn{4}{|c|}{ Degree of lung lesions } \\
\hline & $\begin{array}{l}\text { Non- } \\
\text { diseased }\end{array}$ & Mild & Moderate & Severe & No lesions & Mild & Moderate & Severe \\
\hline $\begin{array}{c}\text { Number of } \\
\text { animals } \\
(n=15)\end{array}$ & 1 & 8 & 1 & 5 & 0 & 3 & 5 & 7 \\
\hline \multicolumn{9}{|l|}{ Pleural fluid } \\
\hline $\begin{array}{l}\text { Min. volume } \\
(\mathrm{mL})\end{array}$ & 1 & 1 & 30 & 15 & - & 1 & 1 & 15 \\
\hline $\begin{array}{l}\text { Max. volume } \\
(\mathrm{mL})\end{array}$ & 1 & 35 & 30 & 230 & - & 13 & 35 & 230 \\
\hline $\begin{array}{l}\text { Mean vol- } \\
\text { ume } \pm \text { SD } \\
(\mathrm{mL})\end{array}$ & - & $11.3 \pm 11.4$ & - & $87.0 \pm 87.7$ & - & $7.0 \pm 6.4$ & $13.8 \pm 13.7$ & $69.3 \pm 77.8$ \\
\hline $\begin{array}{l}\text { Min. cell count } \\
\text { (cfu/mL) }\end{array}$ & 0 & 0 & $4.2 \times 10^{7}$ & $9.7 \times 10^{7}$ & - & 0 & 0 & $4.2 \times 10^{7}$ \\
\hline $\begin{array}{l}\text { Max. cell count } \\
(\mathrm{cfu} / \mathrm{mL})\end{array}$ & 0 & $2.7 \times 10^{8}$ & $4.2 \times 10^{7}$ & $1.3 \times 10^{9}$ & - & $1.5 \times 10^{7}$ & $2.7 \times 10^{8}$ & $1.3 \times 10^{9}$ \\
\hline $\begin{array}{l}\text { Mean cell } \\
\text { count } \pm S D \\
(\mathrm{cfu} / \mathrm{mL})\end{array}$ & - & $5.9 \times 10^{7} \pm 9.7 \times 10^{7}$ & - & $4.1 \times 10^{8} \pm 5.0 \times 10^{8}$ & - & $7.5 \times 10^{6} \pm 8.6 \times 10^{6}$ & $6.4 \times 10^{7} \pm 1.2 \times 10^{8}$ & $3.2 \times 10^{8} \pm 4.4 \times 10^{8}$ \\
\hline \multicolumn{9}{|l|}{ Pericardial fluid } \\
\hline $\begin{array}{l}\text { Min. volume } \\
(\mathrm{mL})\end{array}$ & 5 & 1 & 4 & 4 & - & 3 & 1 & 4 \\
\hline $\begin{array}{l}\text { Max. volume } \\
(\mathrm{mL})\end{array}$ & 5 & 65 & 4 & 70 & - & 18 & 65 & 70 \\
\hline $\begin{array}{l}\text { Mean vol- } \\
\text { ume } \pm \text { SD } \\
(\mathrm{mL})\end{array}$ & - & $13.5 \pm 21.6$ & - & $29.0 \pm 27.5$ & - & $10.5 \pm 8.1$ & $15.6 \pm 27.8$ & $22.6 \pm 25.0$ \\
\hline $\begin{array}{l}\text { Min. cell count } \\
(\mathrm{cfu} / \mathrm{mL})\end{array}$ & 0 & 0 & $1.2 \times 10^{6}$ & $8.7 \times 10^{4}$ & - & 0 & 0 & $8.7 \times 10^{4}$ \\
\hline $\begin{array}{l}\text { Max. cell count } \\
\text { (cfu/mL) }\end{array}$ & 0 & $4.0 \times 10^{5}$ & $1.2 \times 10^{6}$ & $2.0 \times 10^{8}$ & - & 17 & $1.5 \times 10^{2}$ & $2.0 \times 10^{8}$ \\
\hline $\begin{array}{l}\text { Mean cell } \\
\text { count } \pm \text { SD } \\
(\text { cfu/mL) }\end{array}$ & - & $5.0 \times 10^{4} \pm 1.4 \times 10^{5}$ & - & $4.0 \times 10^{7} \pm 8.9 \times 10^{7}$ & - & $8.5 \pm 9.8$ & $32.4 \pm 65.9$ & $2.9 \times 10^{7} \pm 7.5 \times 10^{7}$ \\
\hline
\end{tabular}

that showed no colonisation of the lungs. It was mainly detected in tissues other than the lungs if the animals had a moderate or high-grade score of isolation from lung tissue, too. There was no detection of A. pleuropneumoniae in blood, kidney, tarsal, carpal and meningeal samples from animals with low-grade isolation from lung tissue. For liver and spleen samples only one pig with a lowgrade lung isolation result was culturally positive.

From the eight animals chosen for histological and IHC examination three were euthanized due to exit criteria and five were euthanized at day 7 pi. Two were clinically classified as non-diseased, four as mildly diseased and two as severely diseased. By IHC examination A. pleuropneumoniae was detected in all eight spleen samples (oligofocal within the red splenic pulp), five liver samples (focal to oligofocal, mainly sinusoidal and intravascular), three kidney samples (focal, intertubular, within the renal pelvis and intravascular embolism), two tarsal samples (focal as intravascular embolus), two meningeal samples (focal, leptomeningeal and pachymeningeal) and in none of the carpal samples. A. pleuropneumoniae was detected on the inner surface of the parietal pericardium in five animals (Figure 1). These positive results were obtained from animals euthanized due to exit criteria (liver: 3 pigs; spleen: 3 pigs; tarsal joints: 1 pig; meninges: 2 pigs) as well as from pigs euthanized at day 7 pi (liver: 2 pigs; spleen: 5 pigs; tarsal joint: 1 pig; meninges: 6 pigs). In the kidney samples $A$. pleuropneumoniae was only detected on day 7 pi by IHC. Although the macroscopic examination revealed no gross lesions in other organs than the lung the histological examination revealed a hypertrophy of the Schweigger-Seidel-sheaths and an acute diffuse moderate to severe hyperaemia in all spleen samples, a moderate neutrophilia in five spleen samples and a hypertrophy of the reticulocytes as well as an increased number of apoptotic cells in two spleen samples. Two pigs had a multifocal, acute and mild to moderate purulent hepatitis (Figure 2), one pig showed a 

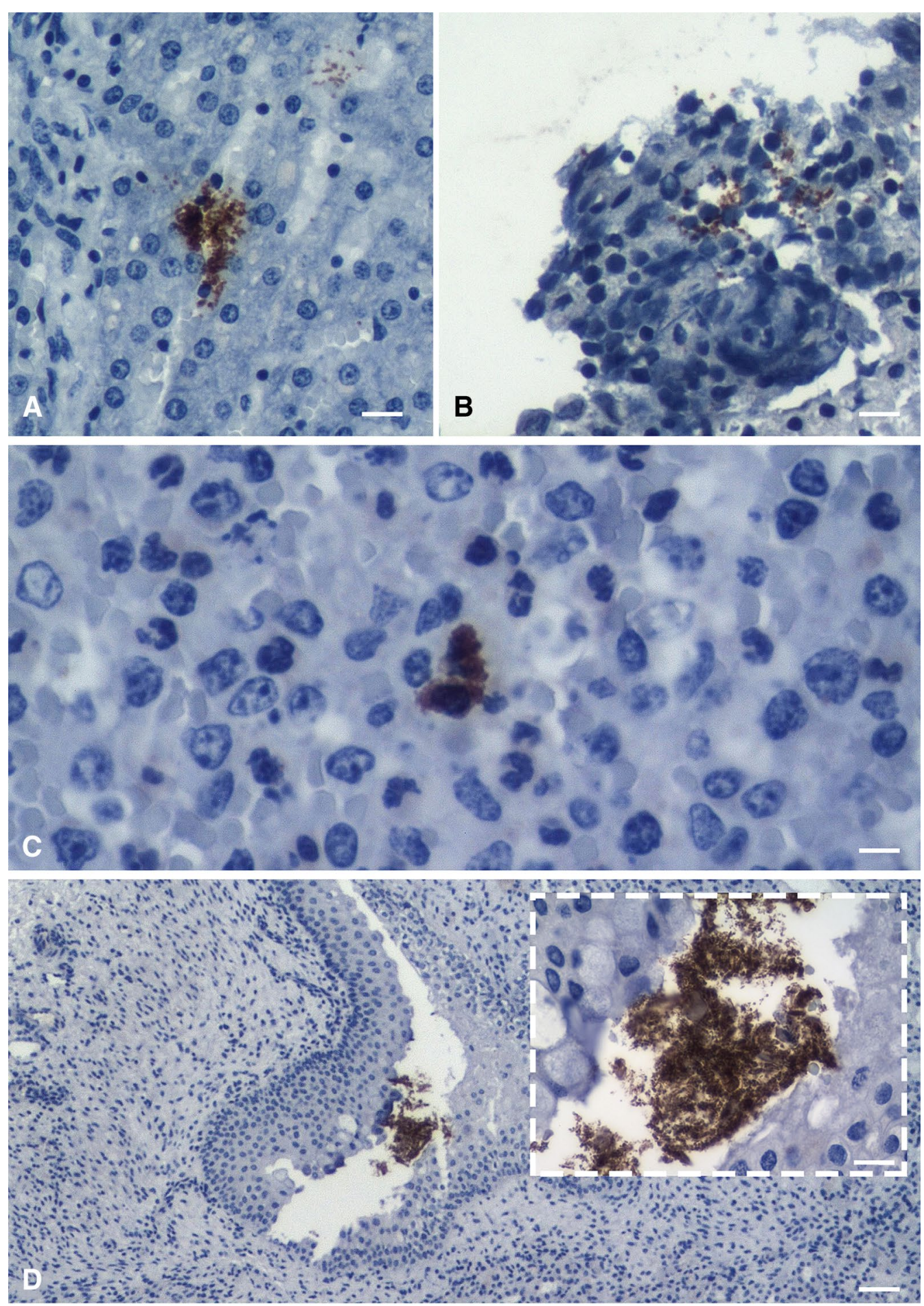

Figure 1 Detection of A. pleuropneumoniae by immunostaining. Detection in different body tissues during acute infection using a Horserad ish-Peroxidase-(HRP)-polymer based detection system with DAB chromogen and Hematoxylin Gill 2 counterstain. A Liver (20-fold magnification): Note oligofocal detection of coccoid bacteria (brown) with A. pleuropneumoniae antigen within sinuisoids. B Pericard (20-fold magnification): Note detection of coccoid bacteria (brown) with A. pleuropneumoniae antigen within superficial exudate containing karyolytic leukocytes some of which are elongated (so called oat cells) which is characteristic of A. pleuropneumoniae. C Spleen (40-fold magnification): Note neutrophilia of the red pulp and detection of A. pleuropneumoniae antigen (brown) within two macrophages. D Kidney (4-fold magnification): Note colony of small rod-shaped bacteria with A. pleuropneumoniae antigen (brown) within the pelvis. Inlet: higher (40-fold) magnification of the bacterial colony. 




Figure 2 Histological lesions in liver and kidney tissue after experimental infection with $A$. pleuropneumoniae. 10 -fold magnification, hematoxylin-eosin staining. A Liver: multifocal, acute, mild to moderate purulent hepatitis B Kidney: focal, acute mild to moderate embolic fibrino-purulent nephritis

focal, acute mild to moderate embolic fibrinous purulent nephritis (Figure 2) and two pigs showed a focal accumulation of neutrophilic granulocytes within Dura mater and cerebrum.

The histological examination of all seven lung samples revealed a severe acute fibrino-haemorrhagic and necrotizing pleuropneumonia for the two pigs that had been clinically classified as severely diseased when chosen for IHC. For two pigs with a mild clinical manifestation nonetheless a severe acute fibrinohaemorrhagic and necrotizing pleuropneumonia was detected but only in the lung tissue sample from the right caudal lung lobe. The third pig with a mild clinical disease showed mild to moderate fibrino-haemorrhagic and necrotizing lung lesions. In lung samples showing necro-haemorrhagic lesions, coccoid bacteria were detected in particular closely related to and within the lymph vessels. The fourth pig with a mild clinical disease and one of the two pigs that had been classified as clinically healthy showed no histological lesions associated with $A$. pleuropneumoniae infection. The second pig that was clinically classified as non-diseased showed a focal acute mild fibrinous pleuritis in the Lobus accessorius including pleural exudate composed of neutrophils, macrophages, fibrin and erythrocytes.

There was no significant correlation, either with the degree of clinical disease or with the degree of isolation from the lungs, the lung lesion score or the detection in other organ samples (Figure 3) for the detection of A. pleuropneumoniae in blood by either method (blood culture or direct plantings).

Volumes of the effusions and A. pleuropneumoniae cfu within the pleural fluids were highly significantly correlated with the degree of clinical disease and the degree of lung lesions (volume: $p^{\text {disease }}=0.005, p^{\text {le- }}$ sions $\leq 0.001$; cell count: $p^{\text {disease }}=0.004, p^{\text {lesions }}=0.001$ ). Regarding the pericardial fluids there was no significant correlation between fluid volumes and the degree of clinical disease or lung lesions developed during acute infection $\left(p^{\text {disease }}=0.264 ; p^{\text {lesions }}=0.129\right)$. However, there was a highly significant correlations between both, the degree of clinical disease and the degree of lung lesions, and the amount of viable A. pleuropneumoniae cells within the pericardial fluids ( $p^{\text {disease/ }}$ lesions $=0.001$ ).

The detection of $A$. pleuropneumoniae by bacteriological culture within the inner organ samples (liver, spleen, kidney) was highly significantly correlated with the degree of isolation from the lung tissue $(p \leq 0.001$; Figure 3 ). The detection in liver and spleen was also highly significantly correlated with the degree of clinical disease (spleen: $p \leq 0.001$; liver: $p=0.003$ ). The same was found for the isolation from spleen tissue and the lung lesion score $(p=0.001)$. For the detection in the kidney and the clinical score $(p=0.013)$ as well as for the lung lesion score and the isolation from liver $(p=0.016)$ and kidney $(p=0.049)$, respectively, there was a significant correlation.

For the peripheral swab samples (carpal joints, tarsal joints and meninges) there was a highly significant correlation between the detection of the bacterium in the meninges and the degree of clinical disease $(p=0.004)$. There was also a significant correlation between the isolation from the lungs and the isolation from tarsal joints $(p=0.032)$ and meninges $(p=0.019)$ as well as for the detection in the meninges and the lung lesion score $(p=0.039)$.

Regarding the isolation of A. pleuropneumoniae from inner organ tissues and peripheral organ swab samples, the isolation was highly significantly correlated between spleen and all peripheral samples (carpal joints: $p=0.010$; tarsal joints: $p=0.004$; meninges: $p=0.004$ ) as well as between tarsal joints and kidney $(p=0.009)$, respectively. 

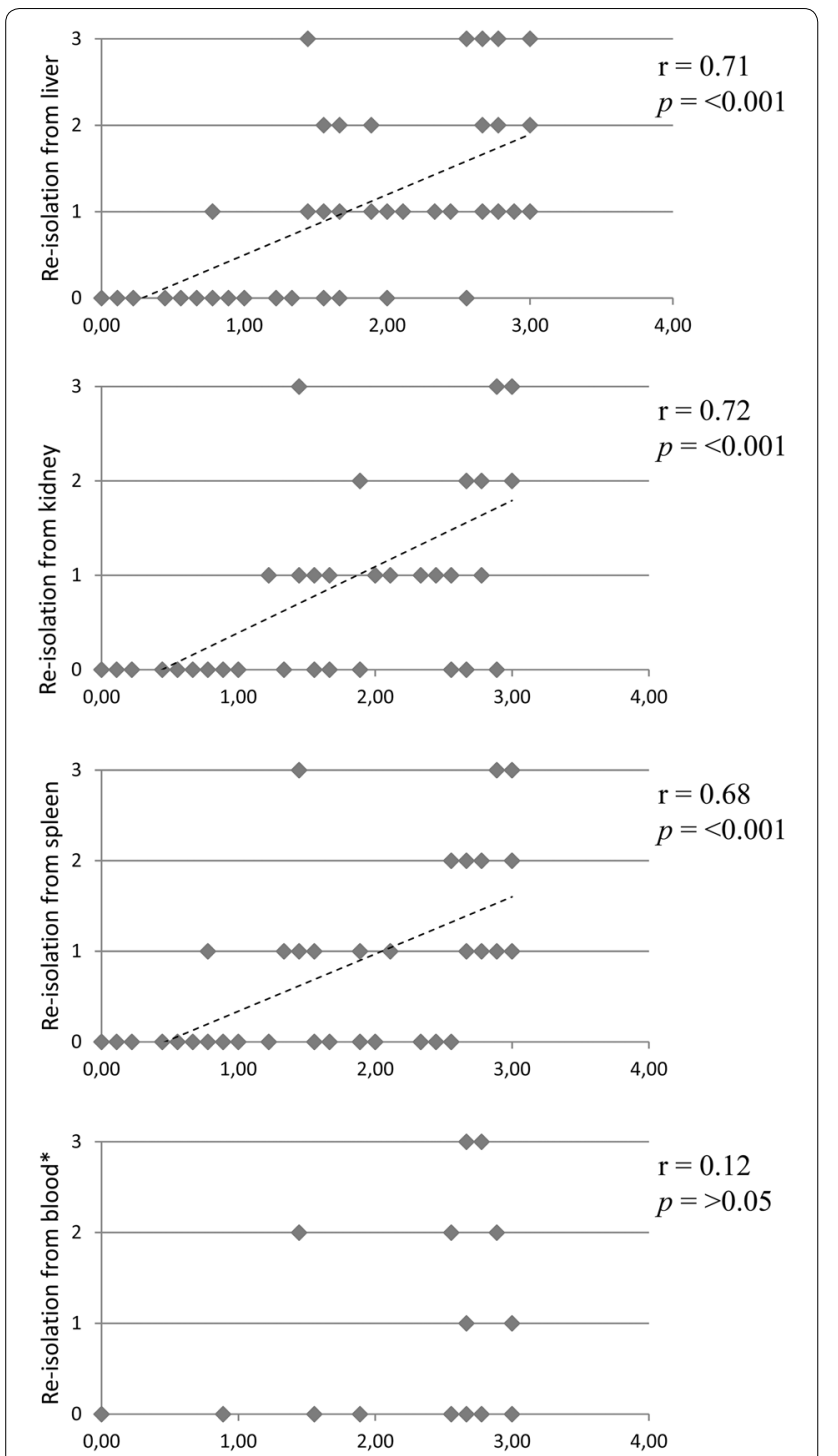

Figure 3 Correlation analysis of isolation from lung tissue and central organ tissues. X-axis: Score quantifying the degree of isolation from seven lung tissue localizations with $0=$ no isolation, $>0-\leq 1=$ low-grade isolation, $>1-\leq 2=$ moderate isolation, $>2=$ high-grade isolation. Degree of isolation from different central organ tissue is displayed on the $y$-axis as isolation score with $0=$ no isolation, $1=$ low-grade isolation, $2=$ moderate isolation, 3 = high-grade isolation; diagonal lines: balance lines for degree of correlation; r: concordance value, $p$ : significance value; concordance and significance calculated by Spearman Rank analysis; * results were obtained by direct plating of blood samples on $A$. pleuropneumoniae-selective blood agar [21] using the quadrant streaking method.

There was a significant correlation between the detection in carpal joint swabs and liver $(p=0.040)$ or kidney $(p=0.018)$ as well as between tarsal joint swabs and liver $(p=0.024)$ and between liver and meninges $(p=0.047)$.

\section{Discussion}

The detection of $A$. pleuropneumoniae in pleural und pericardial fluids of pigs during acute infection are in accordance with earlier studies on porcine pleuropneumonia pathogenesis describing the mechanism of spreading of the pathogen within the pleural cavity not only via lymph vessels but also by oedematous fluids [13] and also describing the incidence of viable bacteria in pericardial fluids in cases of severe pleuropneumonia [27]. Though there was a significant correlation between the degree of clinical disease and lung lesions and detected cfu within the pericardial fluids, we could show, that, in contrast to Nicolet and König [27], A. pleuropneumoniae does not only appear in the pericardial fluids of animals developing a severe disease after infection, but also in the pericardial fluids of pigs only showing mild clinical symptoms and mild lung lesions. Since A. pleuropneumoniae strain AP 76 could be isolated from all tested organ locations, it can be concluded that this strain spreads regularly within the whole body of the pig during the acute phase of infection. Notably, an examination by PCR might have been more sensitive [28] and, thus, might have even revealed higher detection rates in other tissues than the lungs, the isolation by bacteriological culture in the present study demonstrates the viability of the detected A. pleuropneumoniae isolates and avoids that the detection might be based on fragments processed by the immune system [29]. As A. pleuropneumoniae antigen was also detected by IHC intravascular, intralesional, and intracellular within macrophages in different tissue samples, contamination of the samples during the sampling procedure can be excluded. Given that A. pleuropneumoniae was detected within the blood samples both by blood culture and direct plating as well as in intravascular emboli in the IHC examination it can be assumed that the spreading within the organism was due to bacteraemia. Since A. pleuropneumoniae is normally considered to be noninvasive [12] the bacterium might reach the bloodstream due to a damage of the blood vessel endothelium within areas of necrotizing pneumonia. However, in the lung samples of three of the animals examined by IHC and histology no damage of the blood vessel endothelium could be detected although the bacterium was detected within other organ tissues. The fact that there was no positive correlation between the detection in blood and the detection in any other organ tissues might, therefore, also be considered as a hint that the bloodstream might not be the first or only way for spreading. Nevertheless, it should be taken into account that bacteraemia is not always detected by blood culture and that a minimum of three samples per patient are needed to reach a sensitivity of $96 \%$ [30]. The histological detection of the bacteria notably adjacent and inside of the pulmonary lymph 
vessels suggests that spreading takes place mainly via the lymph system. In this case the pathogen might reach the blood stream subsequently via the thoracic duct at the venous angle. In conclusion, it remains to be clarified whether the spreading of $A$. pleuropneumoniae mainly occurs via the lymph system, as stated for the spreading from the lungs to the pleura [13] or mainly by the blood system. Direct passage to the blood stream might also be an incidental event within damaged lung tissue. Nevertheless, the results suggest that pathogenic isolate AP76, in contrast to previous reports on the pathogenesis of A. pleuropneumoniae infection, seems to have an invasive capacity. The histological findings indicate that in most cases during acute infection A. pleuropneumoniae seems to be harboured within different organs without causing any lesions or immune reactions. However, this investigation also reveals mild cases of nephritis and hepatitis, as well as findings of indicative possible beginnings of meningitis. These results are in accordance with the published case reports in which A. pleuropneumoniae was identified as the causative agent of clinical relevant hepatitis [15], meningitis and nephritis [16]. The detection in the tarsal and carpal swabs also might indicate an emigration to the joints even if a minimal contamination of the synovia with blood during sampling cannot be excluded with certainty. However, the fact that the agent was detected in these locations corresponds to a reported case of fibrino-purulent arthritis and necrotizing osteomyelitis [14], although, no alterations could be detected by histological examination of the joint capsules in our samples.

So far, we know hardly anything about virulence mechanisms involved in causing tissue alterations in organs other than lungs or, how long the bacterium survives in these organs and how it escapes the immune system especially during bacteraemia. Combining the IHC results and the results of the correlative analysis it appears that the degree of the colonisation of the lungs, as well, as the colonisation of the spleen seems to play a central role for the spreading of strain AP76 within the pig's organs.

Regarding the results presented here, regularly multiorgan spreading within the pig seems to be proven for at least $A$. pleuropneumoniae serovar 7 strain AP67 used in this experiment. Whether this applies to other isolates and other serovars, too, still needs to be explored. However, the fact, that the strains identified in the published case report on other organ diseases include strains of serovar $2[14,15,17]$, serovar 6 $[16,17]$ and serovar 3 [31] suggests that the ability for spreading within the whole organism of the pig might be held by several $A$. pleuropneumoniae strains.
Actinobacillus pleuropneumoniae strain AP76 seems to spread regularly to different body tissues of the pig during the acute phase of infection. The detection of the pathogen is not only limited to lungs and pleural cavity suggesting that it has invasive capacities. The pathogens ability to colonize the lungs and the degree of spleen colonisation are highly significantly correlated to the extent of spreading within the pig. In most cases A. pleuropneumoniae does not cause pathomorphological alterations in other organs than lungs and pleural cavity. Nevertheless, the strain used within this study seems to harbour the potential of causing lesion within these other organs as in five of eight animals inflammatory lesions were detected by histological examination. Further investigations are needed to examine underlying mechanisms of invasion involved in triggering disease of tissues other than the lungs, as well as, to identify the route of spreading, the ability of other strains and serovars for multi-organ spreading and the survival time of the agent when harboured in other tissues.

\section{Abbreviations \\ A.: Actinobacillus; Ab: antibody; cfu: colony-forming unit; $\mathrm{cm}^{2}$ : square centi- metre; Co.: company; EDTA: ethylenediaminetetraacetate; IHC: immunohis- tochemistry; kg: kilogram; $\mathrm{m}^{2}$ : square meters; $\mathrm{mg}$ : milligram; $\mathrm{mL}$ : millilitre; $\mathrm{NaCl}$ : natrium chloride; PCR: polymerase chain reaction; pi: post-infection; SD: standard deviation; $\mu \mathrm{m}$ : micrometre.}

\section{Competing interests \\ The authors declare that they have no competing interests.}

\section{Authors' contributions}

DH conceived, designed, coordinated and carried out the clinical studies, prepared and carried out the aerosol infection, performed the necropsies, participated in the isolation procedures, performed the statistical analysis and drafted the manuscript. JR and JM participated in the design of the study, carried out the isolation of the bacterium from the post mortem samples of all animals and helped to draft the manuscript. FN participated in the clinical studies and necropsies, participated in the isolation work and helped to draft the manuscript. RF carried out and interpreted the IHC and histology of all pigs entering the study and helped to draft the manuscript. KHW and PVW participated in the design and coordination of the studies. All authors read and approved the final manuscript.

\section{Acknowledgements}

The authors would like to thank the animal caretakers Melanie Rothe and Vanessa Rheinländer from the Clinic for Swine and Small Ruminants and forensic Medicine and Ambulatory Service, University of Veterinary Medicine, Foundation for their support during these studies.

\section{Author details \\ ${ }^{1}$ Clinic for Swine and Small Ruminants and forensic Medicine and Ambulatory Service, University of Veterinary Medicine, Foundation, Bischofsholer Damm 15, 30173 Hannover, Germany. ${ }^{2}$ Institute for Microbiology, University of Vet- erinary Medicine, Foundation, Bischofsholer Damm 15, 30173 Hannover, Ger- many. ${ }^{3}$ Innovative Veterinary Diagnostics (IVD GmbH), Albert-Einstein-Str. 5, 30926 Seelze, Germany. ${ }^{4}$ Clinic for Swine, Department of Veterinary Medicine, Justus-Liebig-University Giessen, Frankfurter Str. 112, 35392 Giessen, Germany.}




\section{Availability of data and materials}

The datasets used and analyzed during the current study are available from the corresponding author on request.

\section{Ethics approval}

The pigs were kept in accordance with the Guidelines for Protection of Vertebrate Animals used for experimental and other Scientific Purposes, European Treaty Series, nos. 123/170. Study design and housing of the animals were approved by a local, independent committee on ethics (Commission for ethical estimation of animal research studies of the Lower Saxonian State Office for Consumer Protection and Food Safety; Approval Number: 33.12-42502-04-15/1962).

\section{Funding}

Parts of this study were supported by the German Ministry of Agriculture (BLE) and the German Annuity Bank (Deutsche Rentenbank).

\section{Publisher's Note}

Springer Nature remains neutral with regard to jurisdictional claims in published maps and institutional affiliations.

Received: 17 July 2018 Accepted: 2 September 2018

Published online: 25 September 2018

\section{References}

1. Gottschalk M (2012) Actinobacillosis. In: Taylor D, Straw BE, Zimmerman $\mathrm{JJ}$, D'Allaire S (eds) Disease of Swine, $10^{\text {th }}$ edn. Wiley, Oxford, pp 653-669

2. Haesebrouck F, Chiers K, Van Overbeke I, Ducatelle R (1997) Actinobacillus pleuropneumoniae infections in pigs: the role of virulence factors in pathogenesis and protection. Vet Microbiol 58:239-249

3. Hoy S, Mehlhorn G, Eulenberger K, Erwerth W, Johannsen U, Dorn W, Hörügel K (1985) Zum Einfluß entzündlicher Lungenveränderungen auf ausgewählte Parameter der Schlachtleistung beim Schwein. Mh. Vet-Med 40:584-587

4. Hoy S, Mehlhorn G, Eulenberger K, Erwerth W, Johannsen U, Dorn W, Hörügel K (1985) Zusammenhang zwischen entzündlichen Lungenveränderungen und der Lebendmasseentwicklung beim Schwein. Mh. Vet-Med 40:579-584

5. Hoeltig D, Hennig-Pauka I, Thies K, Rehm T, Beyerbach M, StrutzbergMinder K, Gerlach G, Waldmann K (2009) A novel Respiratory Health Score (RHS) supports a role of acute lung damage and pig breed in the course of an Actinobacillus pleuropneumoniae infection. BMC Vet Res 5:14

6. Jacobsen M, Nielsen J, Nielsen R (1996) Comparison of virulence of different Actinobacillus pleuropneumoniae serotypes and biotypes using an aerosol infection model. Vet Microbiol 49:159-168

7. Tobias T (2014) Actinobacillus pleuropneumoniae transmission and clinical outbreaks. Ph.D. Thesis, Utrecht University,

8. Gottschalk M (2012) Actinobacillus pleuropneumoniae is a current and continuing pathogen. Albéitar 2:4-5

9. Rycroft AN, Garside LH (2000) Actinobacillus species and their role in animal disease. Vet J 159:18-36

10. Von Altrock A (1998) Occurrence of bacterial infectious agents in pathologically/anatomically altered lungs of pigs and compilation of resistance spectra. Berl Munch Tierarztl Wochenschr 111:164-172

11. Maclnnes J, Desrosiers R (1999) Agents of the "suis-ide diseases" of swine: Actinobacillus suis, Haemophilus parasuis, and Streptococcus suis. Can J Vet Res 63:83-89

12. Bossé JT, Janson H, Sheehan BJ, Beddek AJ, Rycroft AN, Simon Kroll J, Langford PR (2002) Actinobacillus pleuropneumoniae: pathobiology and pathogenesis of infection. Microbes Infect 4:225-235

13. Ajito T, Haga Y, Homma S, Goryo M, Okada K (1996) Immunohistological evaluation on respiratory lesions of pigs intranasally inoculated with Actinobacillus pleuropneumoniae serotype 1. J Vet Med Sci 58:297-303

14. Jensen TK, Boye $M$, Hagedorn-Olsen T, Riising $H$, Angen $\varnothing$ (1999) Actinobacillus pleuropneumoniae osteomyelitis in pigs demonstrated by fluorescent in situ hybridization. Vet Pathol 36:258-261
15. Ohba T, Shibahara T, Kobayashi H, Takashima A, Nagoshi M, Osanai R, Kubo M (2008) Multifocal granulomatous hepatitis caused by Actinobacillus pleuropneumoniae serotype 2 in slaughter pigs. J Comp Pathol 139:61-66

16. Madsen LW, Boye M, Jensen TK, Svensmark B (2001) Actinobacillus pleuropneumoniae demonstrated in situ in exudative meningitis and nephritis. Vet Rec 149:746-747

17. Buttenschøn J, Friis N, Aalbaek B, Jensen TK, Iburg T, Mousing J (1997) Microbiology and pathology of fibrinous pericarditis in Danish slaughter pigs. ZentralbI Veterinarmed A 44:271-280

18. Frey J (2003) Detection, identification, and subtyping of Actinobacillus pleuropneumoniae. Methods Mol Biol 216:87-95

19. StahI WR (1967) Scaling of respiratory variables in mammals. J Appl Physiol 22:453-460

20. Höltig D, Hennig-Pauka I, Beyerbach M, Thies K, Rehm T, Gerlach GF, Waldmann KH, Iras F-K (2008) Comparison of the diagnostic significance of clinical, radiographic and ultrasonographic results after an experimental aerosol infection of pigs with Actinobacillus pleuropneumoniae. Berl Munch Tierarztl Wochenschr 121:422-431 (in German)

21. Hannan P, Bhogal B, Fish J (1982) Tylosin tartrate and tiamutilin effects on experimental piglet pneumonia induced with pneumonic pig lung homogenate containing mycoplasmas, bacteria and viruses. Res Vet Sci 33:76-88

22. Jacobsen MJ, Nielsen JP (1995) Development and evaluation of a selective and indicative medium for isolation of Actinobacillus pleuropneumoniae from tonsils. Vet Microbiol 47:191-197

23. Sachse K, Frey J (2003) PCR detection of microbial pathogens, vol 216. Springer, Berlin

24. Maas A, Jacobsen ID, Meens J, Gerlach G-F (2006) Use of an Actinobacillus pleuropneumoniae multiple mutant as a vaccine that allows differentiation of vaccinated and infected animals. Infect Immun 74:4124-4132

25. Bunka S, Christensen C, Potter AA, Willson PJ, Gerlach G-F (1995) Cloning and characterization of a protective outer membrane lipoprotein of Actinobacillus pleuropneumoniae serotype 5. Infect Immun 63:2797-2800

26. Gerlach G, Anderson C, Klashinsky S, Rossi-Campos A, Potter A, Willson P (1993) Molecular characterization of a protective outer membrane lipoprotein (OmlA) from Actinobacillus pleuropneumoniae serotype 1. Infect Immun 61:565-572

27. Nicolet J, König H (1966) On haemophilus-pleuropneumonia in swine. Bacteriologic, patho-anatomic and histological findings, Preliminary report. Pathol Microbiol 29:301-306 (in German)

28. Gram T, Ahrens P, Nielsen J (1996) Evaluation of a PCR for detection of Actinobacillus pleuropneumoniae in mixed bacterial cultures from tonsils. Vet Microbiol 51:95-104

29. Skovgaard K, Mortensen S, Boye M, Hedegaard J, Heegaard PM (2010) Hepatic gene expression changes in pigs experimentally infected with the lung pathogen Actinobacillus pleuropneumoniae as analysed with an innate immunity focused microarray. Innate Immun 16:343-353

30. Lee A, Mirrett S, Reller LB, Weinstein MP (2007) Detection of bloodstream infections in adults: how many blood cultures are needed? J Clin Microbiol 45:3546-3548

31. Brandreth S, Smith I (1987) Comparative virulence of some English strains of Haemophilus pleuropneumoniae serotypes 2 and 3 in the pig. Res Vet Sci 42:187-193

Ready to submit your research? Choose BMC and benefit from:

- fast, convenient online submission

- thorough peer review by experienced researchers in your field

- rapid publication on acceptance

- support for research data, including large and complex data types

- gold Open Access which fosters wider collaboration and increased citations

- maximum visibility for your research: over $100 \mathrm{M}$ website views per year

At $\mathrm{BMC}$, research is always in progress.

Learn more biomedcentral.com/submissions 